\title{
Application of Vibration Testing Technology in Table Tennis Ball Land Point Location
}

\author{
Meiling Cui \\ Physics and Electronic Engineering \\ College of Harbin Normal University \\ China \\ 363645157@qq.com
}

\author{
Jian Zhang \\ Physics and Electronic Engineering \\ College of Harbin Normal University \\ China \\ 491756966@qq.com
}

\begin{abstract}
Statistics is very important for table tennis (TT) players, coaches, referees, even TT fans. But now it is an extravagant hope because there hadn't been any statistics for a TT match. The basic elements of TT is strength, speed, spinning and landing point. In order to implement the TT landing point location, this paper designs a set of experiments to study the relationship between ball landing and responding of TT table in the circumstances of the same excitation strength and different landing points, different excitation strengths and the same landing point, as well as floor vibration interference etc.. Selects the piezoelectric acceleration sensor to pick up the ball table vibration signal, data acquisition through the oscilloscope, the vibration signals is analyzed in time and frequency domains in Matlab. The achievement laid a sound foundation for accurate locating TT ball landing point.
\end{abstract}

Keywords-table tennis; vibration analysis; passive location

\section{INTRODUCTION}

Table tennis sport is extensively popular in China. The application of table tennis serving machine as an auxiliary device brings many benefits. Instead of coach swing racket personally, the TT serving machine dispenses ball in predetermined way, it is good for athletes to form dynamic stereotype, correct the wrong action, improve footwork and handle etc. and shows remarkable effects. Although serving machine system is perfect, but there is no automatic qualitative and quantitative assessment method. Teaching evaluation is neseccerry in every stage of training. Information acquisition, data processing has been done artificially in training and competition for a long time. That causes sport information processing with low efficiency and low accuracy. But intelligent, digital electronic technology has entered into many sports. Accurate detection problem of table tennis ball landing point becomes one of the main problems in TT information processing. Except the excitation of balls, the table suffers a lot of interference from nature and human being. External interference, for example, player stamp, noise, ball's double-bounce etc., all of this may cause false judgment. Correct distinguish the causes from table response signals are very important. Based on this consideration, an experimental system is designed to collect vibration signal and analyze the signal in both time and frequency domains. Features of the system response are obtained in the case of different excitations.
Further anti-interference measure can be taken to improve the judgment probability.

\section{EXPERIMENT SYSTEM DESIGN}

We divide one side of a TT table into nine sections. Each section is supported as a cantilever and an acceleration sensor mounts down side of each cantilever. Structure of experimental system, which is designed to collect and analyze vibration signal, is shown in figure 1 . The cantilever is made of plastic insulation board which is $500 \mathrm{mmX} 400 \mathrm{mmX} 4 \mathrm{~mm}$. Piezo-electric accelerometer is fixed in the middle of its free end. Its output signal is collected and recorded by an oscilloscope through a precharge amplifier. And the signal is transmitted to a computer through a standard USB interface. The signal is analyzed both in time and frequency domains on computer with Matlab and its characteristics are abstracted.

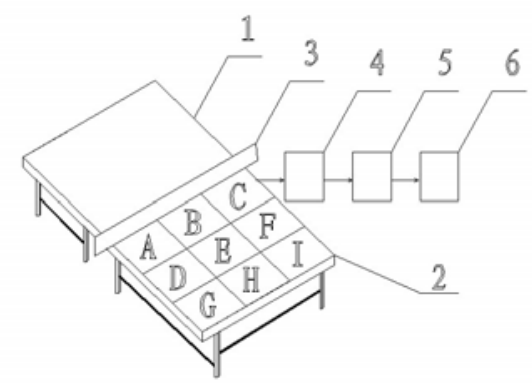

1 Normal Tables 2 Divided Table 3 Net 4 Sensor Preamplifier 5 Oscilloscope $6 \mathrm{PC}$

FIGURE 1. STRUCTURE OF EXPERIMENTAL SYSTEM

Piezo-acceleration probe is selected as a transmitting element for its small size, light, low power consumption and long service time. Above all its dynamic characteristic is favorable. It is widely applied in acceleration measurement, dynamic force, vibration or pressure. The structure of Piezoacceleration probe is simple. It is consist of a mass block, piezo-element and a support. The support is mounted on the cantilever. As an external excitation acting on the cantilever, the acceleration probe vibrates along with the cantilever. Piezo-element is subjected to the inertial force of mass block. According to Newton second law, inertial force is the function of acceleration, that is $F=m a$. The inertial force 
acts on piezo-element, the amount of charge generated is proportional to the acceleration. Passing through a precharge amplifier, shown in figure 2 , the output signal passes to an oscilloscope.

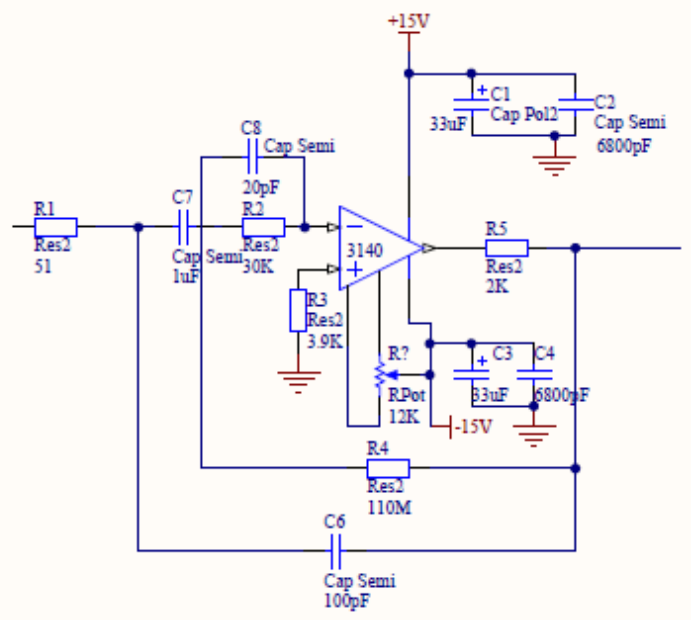

Figure 2. CHARGE AMPLIFIER CIRCUIT

We chose GDS-2202 double trace oscilloscope to do data acquisition and record. Its bandwidth is $100 \mathrm{MHz}$, sampling rate is $100 \mathrm{Msa} / \mathrm{s}$, fifteen group memory can be accessed in front panel, two groups memory are for waveform recording. This series oscilloscope can be remote accessed with a computer via a built in RS-232 interface. And it can transmit data collected to a computer through a USB interface.

\section{EXPERIMENT STUDY}

\section{A. Experiment Process}

The experiment system is for studying the system response to a ping pang ball excitation and a player stamp. Group one experiment is divided into two categories, that is different distance away from but same landing point on the table, same distance away from but different landing point on the table. Second group experiment is focused on interference. Several system response signals of stamp excitation are collected.

In the experiment system, shown in figure 1, the cantilever is knocked by ping pang ball. Cantilever response to knock is transmitted to an electrical signal by piezoacceleration sensor. Taking the sensor position as the original point, the ping pang ball falls freely from $500 \mathrm{~mm}$ and $1500 \mathrm{~mm}$ high and is $100 \mathrm{~mm}$ away from original point at first; then the ping pang ball falls freely from $500 \mathrm{~mm}$ high and is $100 \mathrm{~mm}$ and $350 \mathrm{~mm}$ away from original point. The experiment is divided into four groups, and takes five times measurement for each group. The peizo-acceleration sensor output signals is collected and stored with an oscilloscope. Oscilloscope transmits these data to a computer. Computer records and stores data with FreeWave software tool. Figure 3 shows one of the recorded signal waveform responding to a ball exitation; Figure 4 is one of the waveform respond to stamp one's foot. And the measurement result lists in table 1 .

TABLE I. AT DIFFERENT EXCITATION CONDITIONS SENSOR OUTPUT VOLTAGE AMPLITUDE

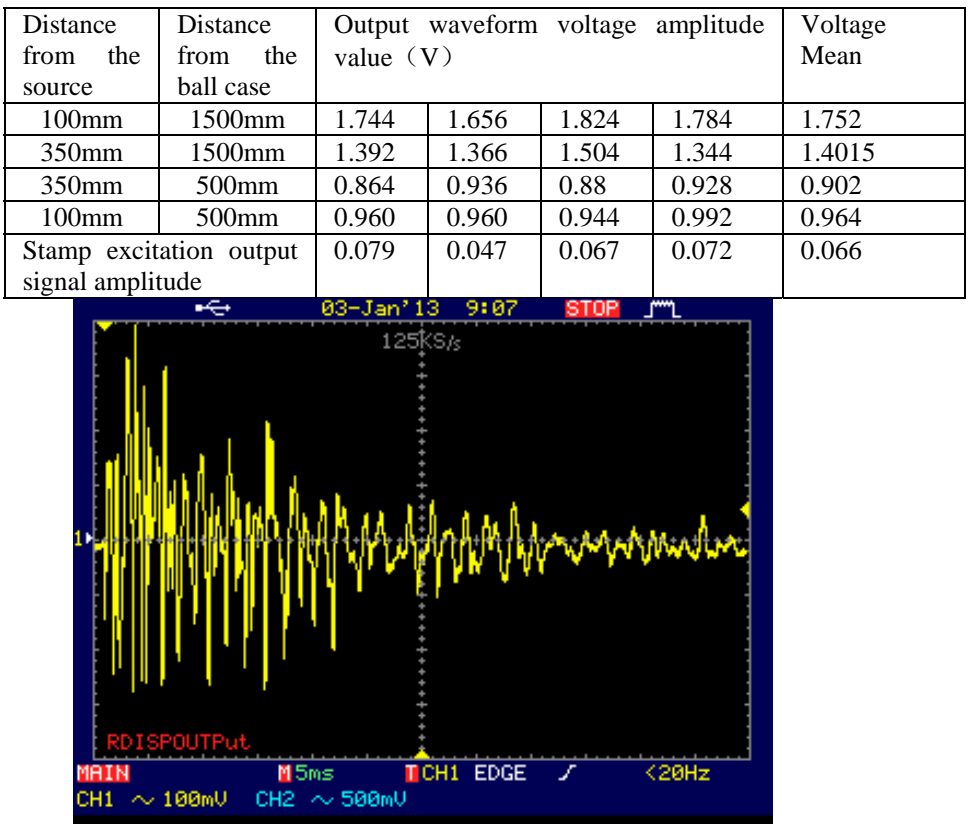

FiguRE 3. BALL RESPONSE SIGNAL

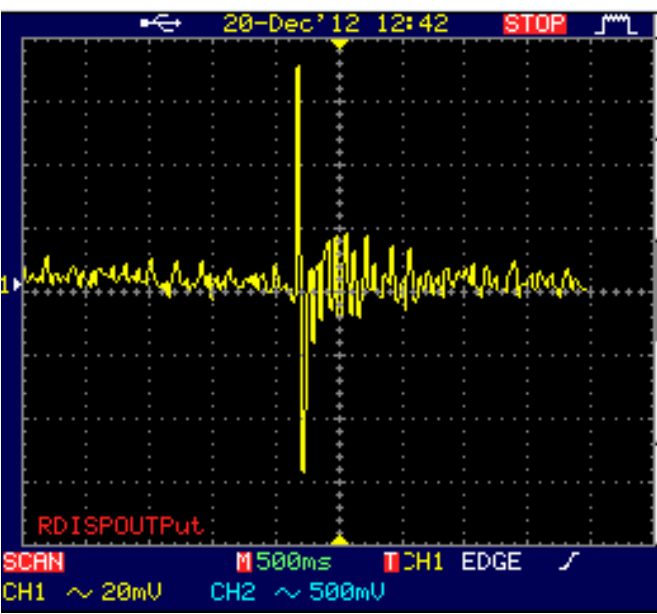

FIGURE4. STAMP RESPONSE SIGNAL

\section{B. Signal Analysis}

a. Time domain analysis

The sensor output amplitude is propotional to cantilever vibration acceleration. The output waveform is damped as time increasing. The experiment data is processed in time domain and listed in table1. Table one shows that:

(1). There are no big differences among the five waveforms obtained in same measurement situation;

(2). The waveform amplitude is different while the measurement situation varies. 
(3). As landing point fixed, the output waveform amplitude is increasing along with the distance increasing between the ball and table; As the distance between the ball and table fixed, the output waveform amplitude is decreasing along with the distance increasing between the landing point and the sensor position.

(4). There is no big influence on output waveform while some interference exists, for example, stamping one's foot. b. Frequency domain analysis

At first, we do not know how much the maximum system response frequency is, so the oscilloscope sample number we select is 25000 . We imported the data recorded in oscilloscope into a computer and stored the data in excel file. Then the data is analyzed via Matlab. Figure 5 shows one of the system response spectrums.

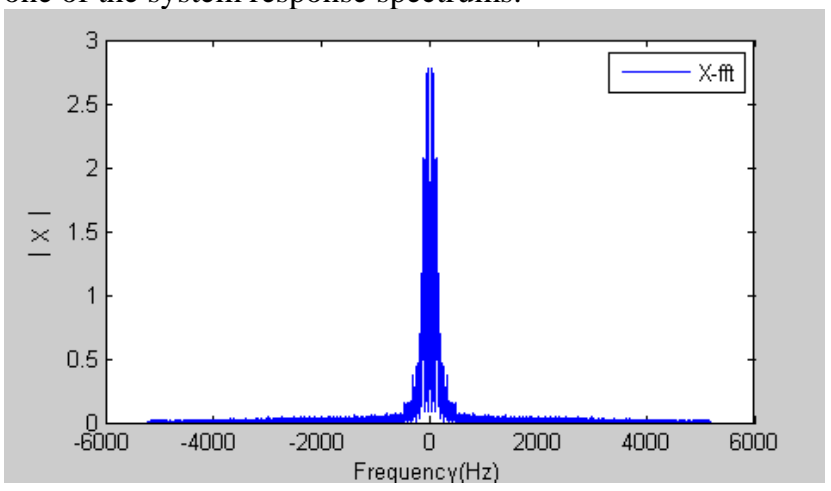

FIGURE 5. SPECTRUM WITH 25,000 POINT SAMPLES

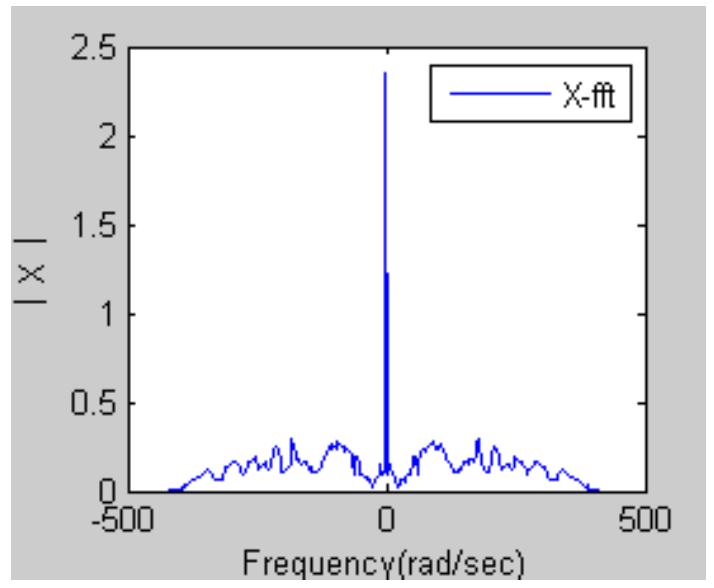

FiguRE 6. SPECTRUM WITH 500 POINT SAMPLES

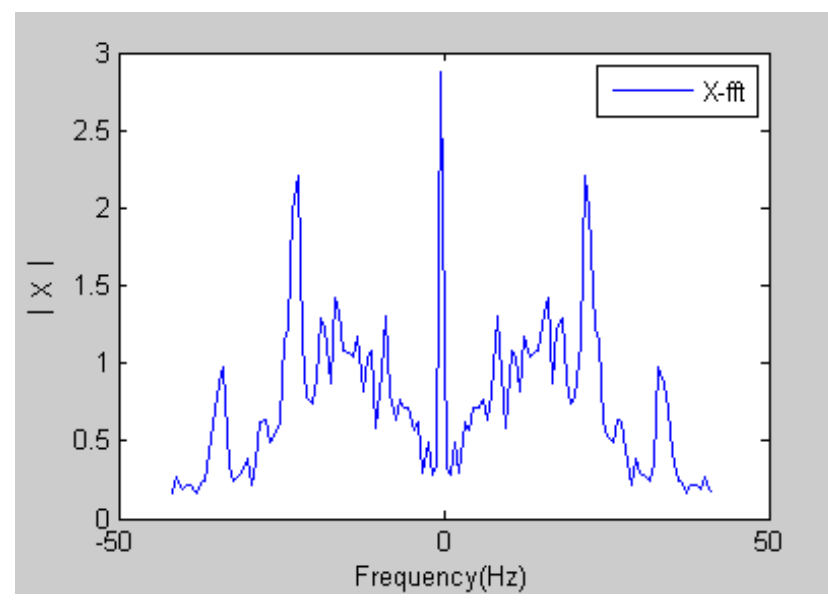

FIGURE 7. STAMP RESPONSE SPECTRUM

Figure 5 shows the system response frequency range is within $500 \mathrm{~Hz}$. In order to have more clearer frequency spectrum, the sample number selected is 500 and one of the system response spectrum is shown in figure 6.

We analyzed all the system response data responding to a ball stipulation and we found that their frequency spectrum distribution is similar and their frequency range is about the same. That is their frequency range is below $400 \mathrm{~Hz}$.

The system response data responding to stamp one's foot are analyzed in the same way. We found that their frequency spectrum distribution is similar and their frequency range is about the same. Their frequency range is below $50 \mathrm{~Hz}$. Figure 7 shows one of the data spectrums.

\section{CONCLUSION}

An experimental test system for TT ball landing point location is proposed in this paper. The peizo-acceleration sensor is applied to pick up the table's vibration, after preamplifier, vibration signals are recorded with an oscilloscope, a computer imported the recorded data and analysis the data by applying FreeWave、 Matlab software. The general rule of TT ball landing characteristics is derived both in time domain and frequency domain. It lays a sound foundation for determining TT ball landing point correctly and precisely. For example, the stamp one's foot interference can be eliminated by a low pass filter and if a comparator's threshold is set properly, some interference may be blocked away.

\section{REFERENCES}

[1] Xiang tao, Based on ADXL330 acceleration sensor vibration sensing applications, Science and Technology Communication,DEC.2010.

[2] Wang wei gao, Vibration signal detection Programmable filter design and application, Technology Innovation Herald, 2012.

[3] Bai ning,Xu xiao ying, Vibration detection method in the screw pump fault diagnosis,JUN.2004.

[4] Yang yu fen, Characteristics of piezoelectric sensors, Information Development \& Economy,2004. 
[5] Li yong jian, Weak Duffing chaotic system based on vibration signal detection method,2009.

[6] Zhang yu fei, College tennis ball machines introduced elective courses to support teaching experimental study, Shenyang Institute of Physical Education,JUN.2009.

[7] Zhong luo jie, Table tennis racket and practicality dedicated training aid Probe, China Sport Science and Technology,2010.
[8] Li yu hua,Zhou yi jie, Tennis different locations after shock speed detection, Jiang Nan University School newspaper,Feb.2013.

[9] Yang hong wei,Zhang yun, Table Tennis rebound trajectory Computer Simulation, Computer simulation,MAR,2006.

[10] Xie feng, On the Table Tennis Teaching in Colleges Evaluation System, zhongzhou tiyu.shaolin yu taiji,SEP,2012. 